28 | 2002

Histoire de l'enseignement du français langue

étrangère ou seconde dans le bassin méditerranéen.

Volume 2

\title{
L'enseignement du français à Lucques de 1805 à 1814 : une expérience novatrice
}

\section{Marie-France Merger}

\section{QpenEdition \\ Journals}

\section{Édition électronique}

URL : https://journals.openedition.org/dhfles/2650

DOI : $10.4000 /$ dhfles.2650

ISSN : 2221-4038

\section{Éditeur}

Société Internationale pour l'Histoire du Français Langue Étrangère ou Seconde

Édition imprimée

Date de publication : 1 juin 2002

Pagination : 71-86

ISSN : 0992-7654

Référence électronique

Marie-France Merger, «L'enseignement du français à Lucques de 1805 à 1814 : une expérience novatrice ", Documents pour l'histoire du français langue étrangère ou seconde [En ligne], 28 | 2002, mis en ligne le 31 janvier 2014, consulté le 27 mai 2021. URL : http://journals.openedition.org/dhfles/2650 ; DOI : https://doi.org/10.4000/dhfles.2650

Ce document a été généré automatiquement le 27 mai 2021.

(c) SIHFLES 


\title{
L'enseignement du français à Lucques de 1805 à 1814 : une expérience novatrice
}

\author{
Marie-France Merger
}

1 Le 15 juillet 1805, l'entrée officielle de la sœur de Napoléon, Elisa Bonaparte et de son mari, Felice Baciucchi - militaire de carrière - à Lucques, marque pour la ville la fin des espoirs de conserver une certaine autonomie. Les Princes réorganisent et restructurent différents secteurs de la vie lucquoise en particulier le domaine juridique et celui de l'instruction publique. En fait, lorsqu'on parle d'instruction et d'éducation ${ }^{1}$ à l'époque des Baciocchi, il est indubitable que le nom d'Elisa apparait, et non pas celui du prince Felice. Dans ce domaine, Elisa aura un rôle de premier plan ${ }^{2}$ : c'est elle qui faisait des remarques sur les règlements, qui supervisait les nominations des enseignants et du personnel administratif, c'est elle qui commandait et on peut penser que, comme le déclarait l'historien Mazzarosa (1833:246) «bien que les décrets fussent au nom du prince, en substance c'était Elisa qui gouvernait l'Etat. »

Notre intention est d'étudier plus précisément l'enseignement du français dans deux établissements d'enseignement secondaire de la « capitale » de la Principauté, des établissements qui ont été créés en 1807 : le collège Félix (collegio Felice), un collège de garçons et l'institut Elisa, un pensionnat pour les jeunes filles.

\section{La création et l'organisation du collège Félix et de I'Institut Elisa}

D'après le décret du 20 novembre 1807, il est interdit d'envoyer les garçons et les filles étudier en dehors de la Principauté, "sous peine d'une amende de 500 francs qui pourrait aller jusqu'à 1000 francs » selon les circonstances ; il en est de même pour les élèves qui se trouvent actuellement dans des établissements étrangers : ils doivent revenir à Lucques avant la fin du mois de mars $1808^{3}$. 
Ce même décret annonce que seront admis au collège Félix, aux frais de l'Etat dix élèves pensionnaires avec une bourse (deux élèves recevront la moitié de la pension, six les deux tiers et deux la pension en entier). Les enfants qui auront eu un prix à l'école primaire auront le droit d'être admis à ce collège selon les places disponibles avec une bourse de deux tiers de la pension. C'est aux enfants de parents «qui auront le mieux servi l'Etat ${ }^{4}$ » que les autres bourses seront attribuées à la suite de la présentation du Gran Giudice, le Grand Juge, c'est-à-dire le Ministre de la Justice chargé de l'Instruction Publique, selon le code Napoléon (31 décembre 1807).

4 Le collège est organisé par le décret du 28 novembre 1807 et prend le nom du prince ; il ouvre le $1^{\text {er }}$ décembre et s'installe, dans un premier temps, dans les locaux du séminaire San Martino où dix-huit chambres ${ }^{5}$ attendent les pensionnaires. Le montant de la pension s'élève à 500 francs (paiement anticipé chaque trimestre) et certains élèves bénéficient d'une bourse pour la totalité ou une partie de la somme. Au 2 mars 1808, huit élèves ${ }^{6}$ ont déjà été admis " par volonté impériale ", deux autres viendront s'ajouter. Il s'agit évidemment d'enfants dont le père occupe une position importante (sénateur, préfet ou officier) dans la Principauté.

5 Un nouveau décret organise d'une manière plus complète le collège (16 février 1809) et, par le décret du 24 février ${ }^{7}$, une Direction Générale de l'Instruction Publique est mise en place avec à sa tête Pietro Cenami. II s'agit d'une reconnaissance de l'importance du secteur éducatif qui, auparavant, faisait partie intégrante, du Ministère de la Justice. Ainsi Cenami et le Grand Juge, L. Matteucci, deviennent les points de référence d'Elisa, d'ailleurs les documents officiels témoignent de leur importance.

6 Le collège devient donc un lycée et est transféré dans les locaux de San Frediano, siège de l'ancienne université laquelle avait été supprimée ${ }^{8}$ Sur la base de la nouvelle législation sept classes sont créées : en septième, les enfants les plus jeunes apprennent à lire, écrire et compter ; c'est en quatrième que l'on commence à étudier le français, tout en continuant l'étude du latin, de l'arithmétique, de l'histoire et de la géographie. En première, les élèves apprennent « la mathématique supérieure » et la physique.

7 Le 7 novembre 1809 est publié un règlement ${ }^{9}:$ il est très précis et très structuré. Selon l'article 10, le nombre des pensionnaires est fixé à trente, et les élèves sont admis de sept à quatorze ans ; ils sont divisés en deux compagnies : les grands et les petits. Le passage de l'une à l'autre est déterminé par l'âge, la grandeur et « à moins que celle-ci ne soit particulièrement importante, des enfants de moins de 14 ans révolus ne peuvent pas être admis dans la Compagnie des grands » (Art.33) ${ }^{10}$.

8 La création de l'institut Elisa par le décret du 2 juillet $1807^{11}$ est « le couronnement de la politique d'Elisa dans le domaine de l'instruction des filles » (Busti : 103). On assiste, en effet, à un véritable tournant : l'État intervient dans un secteur qui appartenait traditionnellement et exclusivement au privé. Comme l'écrivait A. Mazzarosa (1833 : 264), le soin « d'éduquer les jeunes filles, surtout celles de noble condition, à Lucques et partout en Italie, était confié auparavant aux religieuses»; si ces dernières pouvaient certes leur inculquer les « saints principes de notre adorable religion » poursuit-il, il n'en reste pas moins que ces sœurs n'arrivaient pas à leur donner une éducation complète ${ }^{12}$.

9 C'est un institut placé « sous la protection spéciale de l'Auguste Souverained'où il tire son nom » comme l'indique Y Almanacco di corte de 1808 ; seslocaux sont ceux du monastère San Domenico qui avait été supprimé. Il devait être composé de douze 
chanoinesses qui ne faisaient aucun vœu, mais qui prêtaient serment d'obéissance aux Constitutions de l'Institut, tandis que la Directrice prêtait serment « dans les mains de Son Altesse Impériale ${ }^{13} »$.

10 En 1808, la première directrice, dont la charge devait durer six ans, est une Française, Maria Felicita Francesca de Villemagne ; en réalité elle ne reste qu'un an et c'est Maddalena Sancasciani de Pise qui lui succède ; le $1^{\text {er }}$ juin 1811, Camille Eenens (ou Enens) ${ }^{14}$, est nommée et sera remplacée par la suite par Rosa Trebiliani, à la fois directrice et surintendante. Cette charge n'était pas prévue dans le 1er règlement de 1808 mais Elisa étant devenue grande duchesse de Toscane se déplace à Florence ; ne pouvant plus contrôler elle-même la vie de cet établissement, elle nomme sa dame d'honneur, Rosa Trebiliani, une educatrice renommée, une personne de confiance qui sera le lien entre elle et l'Institut.

\section{L'organisation des études et l'enseignement du français au collège Félix}

$11 \mathrm{Au}$ collège Félix, où la plupart des enseignants sont des prêtres, l'éducation que reçoivent les élèves " consistera 1 . dans les cours d'écriture (bel carattere), dessin, musique. 2. latin, italien et français ; éléments de rhétorique, géographie et histoire, 3. logique, métaphysique, éthique, physique et mathématiques ${ }^{15}$ ».

12 A la fin de l'année scolaire prévue pour le 30 septembre, aura lieu la distribution des prix à laquelle assistera la princesse Elisa. Celle-ci ayant été éduquée à l'école de Saint Cyr pense que l'émulation est un facteur important dans l'éducation, tout comme le directeur, C. Lucchesini, qui déclare : " l'honneur est pour l'homme l'une des principales incitations à bien faire ", ainsi les professeurs et tous les responsables ont voulu stimuler "l'émulation de chaque individu à l'égard de ses camarades » au cours de toute l'année scolaire ${ }^{16}$.

13 Le premier professeur de français sera l'abbé Guyot. Malheureusement nous avons très peu de documents sur cet enseignant, mais un fait est sûr : le premier prix n'est pas décerné en français ; seuls trois élèves ${ }^{17}$ ont une mention honorable ; en effet, il faut bien reconnaître qu'en ce qui concerne cette matière la situation n'est pas brillante. En septembre 1808, le directeur envoie un rapport au Grand Juge, donc à Elisa, où il fait état de bien des problèmes : en français il a vu peu de progrès chez les élèves et cette situation est due, selon lui, à deux causes : la première " est que les collégiens sont entrés à des moments différents et il a fallu créer plusieurs classes et recommencer par les premiers éléments pour chaque nouveau venu. " Il est donc indispensable de remédier à cet inconvénient à l'avenir : si un élève est admis au collège sans aucune notion de français, il devra l'être obligatoirement au début de l'année scolaire. La seconde raison du manque de progrès des élèves est « la longueur de la méthode » de l'abbé Guyot, d'ailleurs "Son Altesse » avait déjà remarqué cet inconvénient ; nous pouvons imaginer par là que les élèves ne faisaient pas assez de progrès...

14 Toujours dans le même rapport, Lucchesini déclare qu'il va essayer de convaincre Guyot de changer de méthode mais il avoue qu'il doute de la bonne réussite de ce projet car un professeur qui n'a pas su jusqu'à maintenant " trouver une méthode bonne et brève " aura certainement du mal à en trouver une à présent. Il ajoute que ce professeur a besoin de compréhension puisqu'il a été malade et qu'il a tout perdu « 
dans sa patrie et qu'il n'a pas d'autres moyens de subsistance en dehors de son traitement actuel ${ }^{18} »$.

L'abbé Guyot meurt au début du mois de novembre, au moment de la rentrée, mais nous verrons que les problèmes de l'enseignement du français resteront les mêmes indépendamment de l'enseignant. Pour remplacer Guyot, quatre personnesprésentent leur candidature : les abbés Francesco Martini, lequel enseignele français depuis dixhuit ans, Francesco Serafino Galli, qui t'enseigne depuishuit ans, puis Ferdinando Di Grazia, un père de famille, enfin GiambattistaManetti qui a été au service du Comte de Saint Prié, ambassadeur deLouis XVI à Constantinople, aux enfants duquel il enseignait l'italien, Matteucci ajoutera le commentaire suivant : « les sujets proposéssont tous d'unehabileté suffisante ${ }^{19} \%$. Toutefois, la chaire ne retrouve aucun titulaire avantla réorganisation complète du collège survenue, nous l'avons vu, enfévrier 1809. D'ailleurs, en novembre 1809, Cenami écrit qu'il manque encore le professeur de français et que pour ce cours très intéressant, il n'a « aucune personne à proposer ${ }^{20}$ ». Quelques jours plus tard, le Grand Juge renchérit en écrivant à la Princesse qu'actuellement « il ne se trouve aucune personne qui puisse sans danger répondre aux attentes » de celle-ci. Il ajoute même ce commentaire assez désabusé : il avait pensé « ouvrir un concours accessible aux personnes étrangères, mais [...] d'habitude les hommes d'un certain mérite ne veulent pas s'exposer aux incertitudes d'un concours ${ }^{21}$ ».

16 Entre-temps, au mois de septembre, un certain Filippo Speciotti, professeur d'italien et de français à Massa, avait demandé le poste vacant de français, alléguant le fait qu'il était âgé et indigent. Dans une lettre adressée au Grand Juge, Cenami fait remarquer que ce maître ne sera pas capable d'assumer les cours qui seront " très fatigants en raison de l'affluence des élèves», puisque le collège était ouvert aussi aux externes. En outre, il ajoute que, d'après lui, le professeur de français doit " être véritablement français, originaire, si possible, d'un de ces départements où on parle la langue la plus pure, car même si un Italien peut arriver à en enseigner l'esprit, il est très difficile qu'il parvienne à en enseigner la bonne prononciation ${ }^{22}$ ".

17 La candidature de Speciotti sera écartée ${ }^{23}$ et le 1er décembre 1809, c'est l'abbé Juret ${ }^{24}$ qui est nommé sur le poste vacant. Il jouit d'un traitement annuel de 800 francs ; c'est avec le maître d'écriture, celui qui est le plus payé, puisque les autres enseignants n'ont qu'un traitement de 500 francs. Dès le mois de juin 1810, Juret demandera une augmentation - ou un logement - à l'intérieur du collège, car comme il l'explique dans une lettre à Cenami, il ne peut pas vivre avec un salaire aussi bas. Ni l'augmentation, ni le logement ne lui seront accordés ${ }^{25}$.

Quelle méthode utilisait-il ? À part le fait que sa méthode " ne présente aucun inconvénient ${ }^{26} "$, nous n'avons trouvé aucun document mentionnant les manuels utilisés par ce maître, mais nous avons pu lire le « tableau sur les méthodes [...] des Ecoles Secondaires » d'autres villes de la Principauté ${ }^{27}$. Ainsi nous apprenons que trois ou quatre fois par semaine, jours des cours de français, les professeurs utilisent la Nouvelle Grammaire de Goudar qu'ils complètent par la lecture des Aventures de Télémaque et par des traductions du français en italien. L'un des maîtres souligne le fait qu'il est important de confronter les deux langues afin que les jeunes élèves italiens n'oublient pas leur propre langue. Quant au professeur de Castelnuovo, « maestro delle tre lingue " - italien, latin, français - il ajoute son " traité personnel de prononciation et d'orthographe française » qu'il a tiré « des meilleurs et des plus récents auteurs » et à la 
fin de chaque leçon, il donne des passages « tirés du Spectacle de la nature par Pluche ou d'autres ouvrages français comme Bossuet » à traduire en italien ${ }^{28}$.

Nous pouvons voir que, pour les garçons, l'apprentissage de la langue étrangère s'effectue d'une manière traditionnelle, c'est-à-dire à partir des règles degrammaire comme pour le latin enseigné à travers un lourd apparat grammatical. En outre, les trois enseignants insistent sur l'importance de la traduction, notamment en italien. N'oublions pas qu'à l'époque de la dominationnapoléonienne, l'italien était à égalité avec le français en Toscane (Brunot $1969: 2^{\mathrm{e}}$ partie 25-27). D'ailleurs les règlements des deux établissementsseront imprimés en français avec la traduction en italien en regard ; en effet, une fois nommée grande duchesse de Toscane, Elisa se fera le défenseur del'italien auprès de son frère Napoléon.

Cependant, la personnalité de Juret n'est pas sans susciter quelques plaintes. Eneffet, à la fin de l'année scolaire, en septembre 1811, Cenami rédige un rapportet l'adresse au Grand Juge : s'il constate, d'une part que les élèves sontdociles, qu'ils se consacrent bien à leurs études et que le collège jouît d'une si bonne réputation qu'il n'y a pas assez de places pour satisfaire les demandesqui viennent «tant du Royaume d'Italie que de l'Empire français ", de l'autre, il souhaite que le professeur de français " apprenne à se concilier l'esprit des élèves parce qu'à l'exception des élèves du collège ${ }^{29}$ ", c'est-à-dire les pensionnaires qui sont obligés de suivre ses cours, presque tous les externes ont abandonné l'étude du français. Deux mois plus tard, sur les instances de Matteucci qui lui demande un rapport sur cet enseignant, Cenami soulignera encore une fois que les défauts de Juret sont " l'extravagance et l'irritabilité de son caractère, ses manières brusques » qui font que les élèves sont dégoûtés et qu'il n'y a plus que dix externes à ses cours, alors qu'une matière aussi intéressante devrait attirer un plus grand nombre d'élèves ; ce professeur lui semble donc « peu utile à l'instruction publique ${ }^{30}$ ».

21 En outre, les rapports mensuels du censeur font état des nombreuses absences de ce professeur et de la mauvaise conduite des élèves durant la classe de français ; d'ailleurs Juret lui-même enverra trois rapports en avril 1812 et finira par avouer qu'il n'arrive plus à tenir sa classe : « J'ai l'honneur de vous prévenir que si l'on n'y remédie pas, on ne peut plus vivre en classe du fait du vacarme que font messieurs Baciocchi [...], Giuseppe Pie-raggi, Ronco et quelques autres pour ne pas les nommer presque tous ${ }^{31}$ ». Le Grand Juge s'était déjà inquiété de cette situation puisqu'il avait demandé à Cenami si Juret était « susceptible d'améliorer son système [d'enseignement] et de se rendre vraiment utile à la jeunesse ${ }^{32}$ ». N'ayant pu obtenir la lettre patente obligatoire pour les Français devant enseigner dans la Principauté, Juret démissione en août 1812 et sera remplacé par Renault, préférable du fait de sa " prononciation et de sa connaissance de la langue française»; certes, il n'a pas beaucoup d'expérience (il n'a donné que quelques cours chez des particuliers) mais c'est un « excellent sujet très attaché au service de Sa Majesté Impériale » et il possède « sufficienti talenti » selon la formule consacrée ${ }^{33}$.

Renault (dont le traitement était de 800 francs, comme celui des professeurs de rhétorique, de grammaire supérieure, contre les 1000 francs des professeurs de dessin, de mathématiques et de physique ${ }^{34}$ ) donnera entière satisfaction et restera jusqu'à la chute des Princes. 


\section{Les études à I'Institut Elisa, reflet des principes d'éducation d'Elisa}

En 1808, il n'y a que six chanoinesses sur les douze prévues, parmi lesquelles quelques « dames » françaises ; en 1809 elles étaient dix, neuf en 1812 et trois seulement en 1813. Elles sont aidées, dans un premier temps, par deux maîtresses, la première Giuseppa Zini enseigne la grammaire italienne, l'histoire sacrée et profane, la traduction à partir du français et la mythologie, la seconde, Luisa Adelaide Carthier, donne des cours de grammaire française et de géographie. En 1809 les maitresses sont au nombre de quatre (deux italiennes Zini et Adami et deux françaises Fanny Desperieres et Giustina Montgiry), un an après de nouveau deux ; en 1811, il ne reste que Zini qui abandonne elle aussi l'Institut l'année suivante, elle est remplacée par une maîtresse de français, Genevesia Acciardi et une d'anglais, Angiola Acciardi ${ }^{35}$. Il est intéressant de souligner que le traitement de l'enseignante de français, avec 600 francs annuels, était supérieur à celui des autres maîtresses et de la directrice (500 francs); c'était le « maestro di ballo » (de danse) avec 720 francs qui avait la meilleure rétribution ${ }^{36}$.

Selon l'article 8 du règlement, l'Institut accueille les filles de sept à quatorze «ns, à ce propos, Pietro Cenami fait remarquer qu'il est difficile " d'instruire et de plier aux habitudes convenables des demoiselles déjà trop formées ${ }^{37} »$. Une maitresse est prévue pour dix élèves ; celles-ci "seront partagées en deux la ' : la première se nommera classe verte, les rubans des robes, de la cernirne, du tablier, du chapeau seront de cette couleur. La seconde classe sera nommée classe Nacarat», selon la tradition de Madame de Maintenon ${ }^{38}$.

En 1808, la pension était de 450 francs annuels qui seront réduits à 400 par la suite ; là aussi comme au collège Félix, sont admises dix filles avec des bourses couvrant la totalité ou une partie de la pension. Par le décret du 2 janvier 1808, six demoiselles ${ }^{39}$ sont admises, auxquelles vont s'ajouter quatre autres pensionnaires alors que onze étaient à la charge de leur famille ${ }^{40}$. Il faut constater que leur nombre ne sera jamais très élevé, il oscillera d'un minimum de vingt et une élèves à un maximum de trentequatre ${ }^{41}$.

Quelles sont les matières enseignées ? Les demoiselles apprennent à lire, écrire ; elles suivent des cours de catéchisme, d'arithmétique, de géographie, d'histoire, de français et d'anglais, de dessin, de musique et de danse et elles font « tutte le opere di mano dal cucire sino ai ricami più belli ${ }^{42}$ ", en d'autres termes, des ouvrages de dame. L'organisation des journées est très rigide et très détaillée dans tous les règlements ; dans le premier, les élèves ont cours de français tous les jours de $9 \mathrm{~h} .30$ à 11 heures $^{43}$. En fait, les règlements ne changeront pas tellement l'organisation de l'Institut : dans le dernier règlement de novembre 1811, les cours de français ont lieu trois fois par semaine, de dix heures à midi avec la géographie ; il est d'ailleurs intéressant de remarquer que cette matière était très souvent enseignée en français ${ }^{44}$.

Quelle est la méthode des maîtresses de français et quels livres utilisent-elles ? Il est difficile de répondre, car nous avons bien peu de documents à notre disposition, si ce n'est une longue lettre (en français) de Rosa Trebiliani écrite au lendemain de sa nomination comme surintendante, car elle venait d'être chargée par la Princesse de " former un plan d'études » pour les élèves de l'Institut ; elle répond donc le 12 mai 1809 et elle expose ses idées sur l'éducation des jeunes filles et l'organisation des études, en 
particulier l'étude du français. Elle souligne qu'elle ne s'est pas fiée à ses connaissances imparfaites, qu'elle a " examiné plusieurs livres » et qu'elle a consulté des personnes instruites; elle poursuit en ces termes :

en admettant que l'Instruction des femmes n'ait besoin d'être ni aussi profonde, ni aussi étendue que celle des hommes je n'en crois pas moins qu'il soit necessaire qu'elles soyent instruites jusque à un certain point dans un certain genre de sciences, d'arts d'agrément et d'ouvrages de femmes, qui à toutes indistinctement peuvent convenir. [...] En conséquence j'ai adopté la maxime qu'on doit prescrire aux Elèves l'étude de la langue, de l'histoire, de la Geografie, de l'Arithmétique et de la Morale ${ }^{45}$.

Elle ajoute que " l'étude de la langue est absolument nécessaire vu qu'il est impossible de s'exprimer non seulement avec elegance, mais pas même avec justesse si on ne connoit pas la grammaire ", cependant elle révèle une idée qui était partagée par beaucoup d'éducateurs de l'époque : « considérant la grammaire comme la science de la parole, et la parole comme le moyen d'exprimer les operations de l'esprit j'ai observé que la grammaire ne saurait être un objet d'étude pendant les premieres années de l'enfance. » En outre, elle a " observé encore que comme les règles fondamentales de la grammaire sont les mêmes pour toutes les langues on pourrait avec succès combiner l'étude de la grammaire avec celle de la langue étrangère. " Toujours selon Rosa Trebiliani

on devrait prescrire pour les Demoiselles Italiennes l'étude de la langue française, et pour les Francoises celle de l'Italienne d'autant plus que la langue étrangère servirait de moyen d'Instruction pour l.i maternelle, qu'il faut posseder preferablement à toute autre ; ainsi avant qu'elles soyent en état de parler, et d'écrire la langue et etrangere il faudrait leur faire faire des traductions dans la Langue maternelle.

Pour atteindre cet objectif rien de mieux que d'écrire des lettres, car tout le monde en a besoin.

Cette longue lettre reflète bien les idées de l'époque sur l'enseignement des langues étrangères aux jeunes filles : comme l'affirme Caria Pellandra (1998 : 41), depuis " toujours les filles ont appris le français sans passer à travers un enseignement grammatical rigoureux. » On préconisait donc les méthodes naturelles pour apprendre les langues ; dans la pédagogie féminine, l'enseignement formel de la grammaire venait après, une fois que les langues maternelles et étrangères étaient bien maitrisées et qu'elles pouvaient permettre la réflexion sur la langue, ce qui implique que les demoiselles apprenaient le français sous sa forme orale ${ }^{46}$, avec des maîtresses françaises, le plus agréablement possible ; cela donnait d'ailleurs d'excellents résultats.

31 La publication de quatre règlements en l'espace de quelques années révèle l'intérêt que la Princesse accordait à cet établissement, et montre « une recherche continuelle, une nécessité de souligner de nouvelles exigences au fur et à mesure que les situations évoluaient et que les problèmes se présentaient » (Busti 1986 : 276).

De toute façon, dans tous les règlements se déroule un fil conducteur, celui de l'émulation, auquel fait allusion la surintendante dans la lettre que nous avons citée. En effet, le cursus des études est caractérisé par des critères de mérite avec une série ininterrompue d'épreuves et de récompenses comme le démontre l'article $75 \mathrm{du}$ dernier règlement :

Les fautes moins graves de conduite ou d'inapplication des Elèves sont notées chaque jour sur l'état des bonnes et des mauvaises marques, et punies à la fin de 
chaque mois par l'inscription de leurs noms sur le tableau des classes, avec l'annotation convenable.

L'Elève qui aura douze marques de mauvaise conduite ou d'inapplication sur plusieurs genres d'étude ou d'ouvrage, sera placée au bas du tableau sous le titre d'insubordonnée ou de paresseuse ${ }^{47}$.

D'ailleurs, Elisa commente elle-même, dans la marge, une lettre où le directeur de l'Instruction publique avait osé écrire que les règles disciplinaires ne devaient pas être trop sévères : « je vous envoyé le règlement intérieur de la maison Caroline de Naples. Vous i verrez que les Délies sont élevées avec plus de rigueur que celles de l'Institut ${ }^{48}$ ", et elle ajoute à propos des marques :

je trouve bien qu'on donne de mauvaises marques nous étions convenus qu'on ne donnerait pas de bonnes marques car les Délies devant faire leur devoir on ne peut pas tous les jours louer ce qu'on est obligé de faire et on doit punir lorsqu'on manque à ce devoir ${ }^{49}$.

A plusieurs reprises, Elisa sera informée que, dans l'Institut, les jeunes filles recevaient une éducation bigote ou presque, c'est pourquoi, déjà le 26 septembre 1810, elle écrit personnellement à Cenami une lettre où elle critique explicitement les pratiques religieuses imposées aux pensionnaires, et conclut en ces termes :

les demoiselles de l'Institut étant destinées à vivre dans le monde, je veux qu'on leur donne des leçons de morale, qu'on leur apprenne à devenir de bonnes mères, des épouses vertueuses, et non qu'on les élève comme si elles devaient passer leur vie dans un couvent ${ }^{50}$.

C'est un point sur lequel Elisa insistera toujours, et qui correspond à sa vision du rôle de la femme dans une société où une formation adéquate de la jeunesse était nécessaire, y compris l'éducation des femmes, " pivot de la solidité de la famille selon l'éthique bourgeoise " (Franchini 1993 : 53). C'est également ce qu'elle pensait à propos de l'éducation des garçons au collège Félix :

j'ai voulu élever l'ame de ces jeunes gens en faire des sujets dévoués à la Patrie soit dans les administrations soit dans le militaire et non des moines ${ }^{51}$,

répètera-t-elle auGrand Juge encore en 1813, peu avant la fin de la Principauté des Baciucchi.

Le passage de l'ancien au nouveau régime commençait à peine à se consolider lorsque le congrès de Vienne place Maria Luisa de Bourbon à la tête du nouveau duché de Lucques, il n'en reste pas moins que dans le secteur de l'instruction Elisa a marqué son époque. Elle a ouvert la voie à la sécularisation de l'enseignement : elle fonde, en effet, des établissements laïcs, en particulier l'Institut Elisa qui est calqué sur l'original français mais qui va devenir le nouveau modèle de l'éducation féminine, avec une pédagogie toute « féminine " pour l'apprentissage du français. Maria Luisa se limitera à changer les noms des deux établissements, et si l'on parcourt le règlement du 23 mai 1819 de l'Institut « Maria Luisa » (ex-Institut Elisa) dont la directrice sera toujours Rosa Trebiliani, il est facile de s'apercevoir à quel point l'œuvre d'Elisa Baciucchi à Lucques, surtout en ce qui concerne l'instruction des filles, revêt une valeur qui dépasse largement les changements de gouvernement. 


\section{BIBLIOGRAPHIE}

\section{Sources d'archives: Archivio di Stato di Lucca}

Almanacco di Corte 180818091812 Lucca, presso Francesco Bertini Stampatore di S.A.l.

Bollettino officiale delle Leggi, e decreti del Principato Lucchese Gran Giudice

Liceo Reale e Pubblica Istruzione du $\mathrm{n}^{\circ} 1^{*}$ au n ${ }^{\circ} 9^{*}$ Segreteria di Stato e di Gabinetto del Principato di Lucca

AUBERT, Françoise (1999): Travaux d'élèves à l'exposition de Paris 1900. Les devoirs des cours de langue et littérature françaises d'un institut florentin : la Santissima Annunziata. Bologne, CLUEB.

\section{Sources secondaires}

BANDINI, Gianfranco (1998) : Il salterio, la Santacroce e l'alfabeto L'istruzione primaria nello Stato di Lucca nella prima metà dell'Ottocento (1805-1847). Florence, Casa Editrice Le Lettere.

BUSTI, Laurina (1984) : «La pubblica istruzione a Lucca durante il periodo baciocchiano » in Catalogo della mostra // Principato napoleonico dei Baciocchi (1805-1814). Riforma dello Stato e società, Lucca, Museo di Palazzo Mansi, 9 giugno-11 novembre 1984, 95-117. Lucques, Nuova grafica lucchese.

BUSTI, LAURINA (1986) : «L'istruzione femminile a Lucca nel periodo baciocchiano » in Ilprincipato napoleonico dei Baciucchi (1805-1814), Riforma dello Stato e società, Atti del Convegno internazionale, 275-289. Lucques, Maria Pacini Fazzi editore.

COLOMBINI MANTOVANI, Adriana (2000) : « Il Collegio Reale delle fanciulle di Milano e i suoi primi maestri di francese ", in MINERVA éd. (2000), 107-121.

FRANCHINI, Silvia (1993) : Élites ed educazione femminile nell'Italia dell'Ottocento. L'Istituto della SS. Annunziata di Firenze. Florence, Olschki.

LAZZARESCHI, Eugenio (1983) : Elisa Buonaparte Baciocchi nella vita e nel costume del suo tempo a cura di Italo Pizzi. Lucques, Maria Pacini Fazzi editore.

MAZZAROSA, Antonio (1833) : Storia di Lucca dalla sua origine fino al MDCCCXIV. T.II. Lucques, Tipografia Giuseppe Giusti.

MINERVA, Nadia éd. (2000) : Dames, Demoiselles, Honnêtes Femmes. Bologne, CLUEB.

PELLANDRA, Carla (1998) : «1780-1880 : De l'universalité aux résistances nationales » in Histoire de la diffusion et de l'enseignement du français dans le monde, Le Français dans le monde, numéro spécial 24-46. Paris, Hachette.

TREBILIANI, Maria Luisa (1992) : Studi storici lucchesi. Personaggi, avvenimenti, società nel XIX secolo. Lucques, Maria Pacini Fazzi.

\section{NOTES}

1. Plusieurs décrets (septembre 1805-28 novembre 1807 et enfin, le plus important, celui du 16 février 1809) organisent tout le secteur de l'enseignement primaire et secondaire. À ce sujet nous 
renvoyons aux travaux de L. Busti et à l'ouvrage de G. Bandini (cf. bibliographie); Elisa fait introduire de nouvelles règles pour l'administration de l'État qui permettent de doter l'instruction publique d'instruments de contrôle plus efficaces. A la fin de la domination française, l'enseignement primaire (lire, écrire et compter) constituait déjà une présence significative dans la plupart des communes et environ 300 maîtres couvraient le territoire.

2. Signalons qu'Elisa Bonaparte est une ancienne élève de la prestigieuse école de Saint-Cyr, un institut créé en 1685 par Madame de Maintenon, laquelle persuada Louis XIV d'ouvrir un institut d'éducation pour 250 filles de gentilhommes pauvres qui pouvaient démontrer quatre quartiers de noblesse. En outre, nous avons trouvé dans un carton de lettres (qui n'ont pas été enregistrées officiellement) que le 25 janvier 1809, depuis Paris, l'Intendant Général de la Maison de Son Altesse Impériale avait envoyé à Elisa le livre intitulé L'Esprit de l'institut des filles de Saint Louis par Madame de Maintenon, preuve supplémentaire et indéniable - à nos yeux - que la Princesse s'intéressait à ces questions d'éducation (Segreteria di Stato e di Gabinetto n. 81 fase. I).

3. Bollettino officiale delle leggi, e decreti del Principato lucchese t.V pp. 346-347. Ainsi un certain Nicolao Controni qui avait demandé une autorisation pour que son fils puisse continuer ses études dans un collège de Pistoia se la voit refuser. (Gran Giudice 122, protocollo 1353, 12 mai 1808). Sauf indication contraire, nous traduisons les textes législatifs et administratifs qui sont publiés en italien.

4. Art. 7, ibid.

5. Gran Giudice désormais G.G. 118, prot. n.452, 11 fév. 1808.

6. G. G. 119 , prot. n. 627,2 mars 1808 .

7. Bollettino officiale..., t. Vili, p. $61 .^{8}$

8. G. G. 139 , prot. n. 930.

9. Bollettino..., t. IX, pp. $87-132$.

10. Il faut remarquerqu'à partir de cette date des externes sont admis aux cours. En 1810, il y avait 21 pensionnaires et 134 externes ; en 1811, 22 pensionnaires et 134 externes (G.G. 226, prot. 3534, 22 avril 1811).

11. Bollettino..,, t. V, n.2, 2-9.

12. C'est l'époque où vont naître en Italie (à Milan, Vérone et Naples) les établissements publics pour lesfilles (les educandati) qui s'inspirent de la structure administrative et pédagogique de la Maison Impériale d'Ecouen. (cf. Colombini Mantovani 2000).

13. Ibidem.

14. Il s'agit de Camille Rafeau, veuve du hollandais ou flamand Eenens, laquelle deviendra "inspectrice" de la Maison Royale de Saint Denis, créée par Napoléon puis directrice de l'Institut SS. Annunziata de Florence en 1821 (cf. S. Franchini $1993: 40$ et F. Aubert 1999 : 17)

15. Cf. Bollettino..., t.V, p. 357.

16. G. G. 130 , prot. n. 2842,21 sept. 1808.

17. II s'agit de Carlo Frediani, Fortunato Fournier et Lelio Guinigi.

18. G. G,130, prot. n.2929, 27 sept. 1808.

19. G. G. 133 , prot. n. 3431,25 nov. 1808 .

20. Liceo Reale 6*, n. 106, 13 nov. 1809.

21. Segreteria di Gabinetto 11, fase. VI, p. 230.

22. G. G. 143, prot. n.1670, 20 sept. 1809.

23. On retrouvera Speciotti, âgé de 71 ans, comme professeur d'italien dans le nouvel établissement créé en 1812 pour les filles des employés et des artisans, la Congrégation de San Felice.

24. Bollettino..., t. IX, pp. 139-140.

25. Liceo Reale $1^{*}$, n. 185, 16 juin 1810.

26. Ibid., n. 419, 14 déc, 1811. 
27. Il s'agit de Camaiore (l'abbé D. Bonuccelli), Castelnuovo Garfagnana (prof. G. Battista Andreoli) et Massa (prof. D. Nardini) Liceo Reale 5*, n. 112, 28 août 1810; ibid., n, 141, 12 oct, 1810, ibid. $7^{*}$, n.6,janv. 1812.

28. Ibid. $5^{*}$, n, 141, 12 oct. 1810.

29. Liceo Reale $1^{*}$, n. 384,28 sept. 1811.

30. Ibid. $1^{*}$, n.419, 14 déc. 1811.

31. Ibid. $7^{*}$, n. 63, 30 mai 1812.

32. Ibid. $6^{*}$, n. 166,5 déc. 1811 .

33. Segreteria di Stato... 122, n. 210,30 oct. 1812. On se contentait de cette " habileté suffisante ", les souverains étant beaucoup plus soucieux de l'obéissance de ces enseignants, du contrôle que l'État pouvait exercer sur eux que de leurs réelles capacités (cf. Bandini $1998: 52$ ).

34. «G.G.386, n. 565, 28 janv. 1814.

35. Almanacco di corte 1808, 1809, 1812.

36. Segreteria di Stato e di Gabinetto 94, fasc. VI, p. 9, s. d. mais 1810.

37. Liceo Reale $3^{*}$, n. 1428 , avril 1809.

38. G.G.117, prot. 222, 21 janv. 1808. Dans le décret, il est spécifié que tout ce qui appartient à l'uniforme des Chanoinesses et des pensionnaires doit être de "manufacture de la Principauté » (Bollettino..., t. V, p. 7).

39. Il s'agit d'Isabella Trebiliani (fille d'un sénateur et de la surintendante), Luisa et Cristina Giorgini (filles du préfet), Lilla Simeon, Lastenia Sonolet ; Maria Giannini (fille d'un sénateur) (Bollettino..., t. VI, p. 5-6).

40. G.G. 117, n. 290, 26janv. 1808.

41. En 1814, cf. Segreteria di Stato 148, fascicolo II, p. 186.

42. Bollettino, p. 5 («tous les travaux d'aiguille jusqu'aux broderies les plus belles»).

43. G. G. 117, n. 222, 21 janv. 1808.

44. Dans un carton de documents non enregistrés de 1812, nous avons trouvé une liste de livres envoyés à la directrice de l'Institut ; parmi les ouvrages, se trouvent deux exemplaires des Elemens de la grammaire française par Lhomond et un Abrégé de la géographie de Crozâi ; cette note est signée de la main de Villemagne, ce qui nous fait supposer que le classement n'est pas exact, puisque Villemagne était directrice en 1808.

45. Segreteria di Stato n. 74, prot. 547, fase. III, 12 mai 1809. Nous respectons l'orthographe de l'original ainsi que celle d'Elisa.

46. Ce sera également la méthode appliquée à l'Institut SS. Annunziata à Florence, la méthode naturelle de Charles de Lasteyrie (cf. Aubert : 19 et suiv.).

47. Règlement général de l'Institut Elisa p. 41 in C. G. 256 n. 958312 nov. 1811.

48. Liceo Reale $4^{*}$ n. 3630 mai 1809.

49. Ibidem

50. Liceo Reale $5^{*}$ n. 12526 sept. 1810.

51. G.G. 383, Note particolari, 8 janv. 1813

\section{RÉSUMÉS}

Notre propos est d'étudier l'enseignement du français dans deux instituts de la « capitale » : le Collegio Felice, une école pour les garçons, organisée par le décret du 28 novembre 1807, et 
l'Istituto Elisa créé par le décret du 2 juillet 1807. Les sources consultées nous livrent le nom des professeurs, leur salaire, etc. L'œuvre accomplie apparaît originale et sera poursuivie sous la Restauration : en effet, si on parcourt le règlement de l'Institut Maria Luisa du 23 mai 1819, il est facile de s'apercevoir à quel point l'œuvre d'Elisa Baciucchi, surtout en ce qui concerne l'instruction des filles, a une valeur qui dépasse les changements de gouvernement.

Our plan is to study the teaching of the French language in two institutions in Lucca : the Collegio Felice, a school for boys, organised by the decree of November 28th 1807 and the Istituto Elisa, created by the decree of July 2nd 1807. The sources consulted have provided us with teachers' names and salaries. The work done seems original and was continued under the Restoration with Maria Luisa ; it is easy to see how far the work of the Princess has a value beyond any change in government.

\section{INDEX}

Keywords : Teaching of French, French as a foreign language, Collegio Felice, Istituto Elisa, Elisa Baciucchi, Italy, XIXth century

Mots-clés : Enseignement du français, FLE, Collegio Felice, Istituto Elisa, Elisa Baciucchi, Italie, XIXe siècle

\section{AUTEUR}

\section{MARIE-FRANCE MERGER}

Université de Pise 